\title{
Batimetría derivada por satélite (sdb): una aproximación a la cartografía batimétrica con imágenes multiespectrales en aguas poco profundas de Bahía Solano, Colombia
}

\section{Satellite-derived bathymetry (SDB): an approach to bathymetric cartography with multispectral images in shallow waters of Bahía Solano, Colombia}

\author{
Mauricio Alejandro Perea-Ardila ${ }^{1}$ y Fernando Oviedo-Barrero ${ }^{1}$
}

\begin{abstract}
RESUMEN
La medición de las profundidades del océano juega un rol fundamental en la planificación y la gestión de recursos marinos y la navegación segura de embarcaciones. La Batimetría Derivada por Satélite (SDB) se presenta como una técnica complementaria para determinar las profundidades en aguas costeras a través de técnicas de Teledetección y Sistemas de Información Geográfica (SIG). El objetivo de este estudio fue determinar la aplicabilidad de la SDB en aguas poco profundas en el sector de "Punta Luna" en bahía Solano, al norte del litoral Pacífico colombiano que utiliza imágenes del satélite Landsat 8 de enero del 2017 y datos batimétricos in situ de noviembre del 2016. El principal resultado obtenido en este estudio fue la estimación de profundidades de hasta $\pm 7 \mathrm{~m}$ con un $\mathrm{R}^{2}=0.80$, se obtuvo un error RMSE y MAE de 1.49 y 2.22 m respectivamente; las profundidades estimadas por la SDB cumplen en un $51.17 \%$ la medida de Incertidumbre Vertical Total (TVU) para la categoría del Orden Especial, referente al estándar de levantamientos hidrográficos de la Organización Hidrográfica Internacional (IOH). Los resultados obtenidos servirán como caso práctico para obtener profundidades mediante imágenes multiespectrales y denotan un referente para los servicios hidrográficos y académicos interesados en temas de investigación marina y costera de la región.
\end{abstract}

Palabras Clave: Batimetría, Landsat 8, Pacífico colombiano, satélite, SIG

\section{ABSTRACT}

Ocean depth measurement plays a fundamental role to plan and manage marine resources and safe boat navigation. Satellite-Derived Bathymetry (SDB) is presented as a complementary technique to determine coastal water depth through remote sensing tools and Geographic

1 Centro de Investigaciones Oceanográficas e Hidrográficas del Pacífico - CCCP. Área de Manejo Integrado de Zona Costera. Capitanía de puerto de Tumaco. Tumaco, Colombia.mperea@dimar.mil.co, mapereaa@ut.edu.co* ORCID: https://orcid.org/0000-0003-4561-0251; ORCID: https://orcid.org/0000-0001-5559-1504 
Information Systems (GIS). The goal of this study was to determine the applicability of the SDB method in shallow waters in the Punta Luna sector in Bahía Solano, northern Colombian Pacific coast, using Landsat 8 satellite images from January 2017 and in situ bathymetric survey data from November 2016. The main result obtained in this study was a depth estimate of up to $\pm 7 \mathrm{~m}$ with $\mathrm{R}^{2}=0.80$, as well as an RMSE and an MAE equivalent to 1.49 and $2.22 \mathrm{~m}$, respectively. Depth estimates obtained using SDB meet $51.17 \%$ of the Total Vertical Uncertainty (TVU) for the Special Order category, regarding the Standards for Hydrographic Surveys from the International Hydrographic Organization (IHO). Results obtained will serve as a reference to calculate depth using multispectral images and a benchmark for hydrographic officials and academics interested in coastal and marine research in the region.

Keywords: Bathymetry, Colombian Pacific, Landsat 8, Satellite, GIS

\section{INTRODUCCIÓN}

Estimar la batimetría del océano juega un rol importante en actividades marítimas y costeras, como la pesca, la gestión portuaria, el tendido de tuberías, la acuicultura entre otras ; también, es de suma importancia para la realización de cartas hidrográficas empleadas en la navegación de embarcaciones y para estudiar los cambios presentados en el fondo marino (Pushparaj \& Hegde, 2016). Los métodos tradicionales para la medición del fondo del mar suponen el uso de técnicas acústicas donde buques hidrográficos albergan dispositivos tecnológicos conocidos como ecosondas, estas pueden ser del tipo monohaz (Single-Beam Echo Sounder) o multihaz (Multi-Beam Echo Sounders), las cuales a partir de pulsos (sonar) capturan datos del fondo marino y estos son registrados e interpretados por medio de programas informáticos especializados. Este método tradicional determina la profundidad y la topografía del lecho marino (Pushparaj \& Hegde, 2016; Ashphaq, 2018). Aunque, esta técnica permite obtener de forma precisa de los perfiles de profundidad a una escala muy detallada, el método está restringido en aguas poco profundas, además, de tener un elevado costo operativo (Gao, 2009).

\section{Batimetría Derivada por Satélite - SDB}

Desde la década de 1970 se realizaron gradualmente los primeros avances en estudios de aplicación de los sensores remotos ópticos para la estimación de la profundidad en aguas costeras (Deng et al. 2008); trabajos pioneros como los de (Polcyn et al. 1970; Lyzenga, 1978; Warne, 1978; Lyzenga, 1985; Philpot, 1989; Ibrahim \& Cracknell, 1990) demostraron en gran medida atención por el uso de estas tecnologías en la aproximación de métodos para la estimación de la profundidad y su aplicación en varios campos de la ciencia. 
La Batimetría Derivada por Satélite (SDB, por sus siglas en inglés) se presenta como una alternativa para la estimación de las profundidades en aguas costeras debido al potencial de los sensores remotos ópticos en capturar información de grandes extensiones de forma continua; en este contexto, la SDB permite caracterizar y monitorear en el tiempo, los cambios ocurridos en el fondo marino (Jagalingam et al. 2015; IHO-IOC, 2019). El uso de los Sistemas de Información Geográfica (SIG) y las técnicas de Teledetección se han vuelto de uso frecuente debido a la eficiencia y rentabilidad que representan para cartografiar batimetría en amplias zonas costeras (Gao, 2009; Putri et al. 2018); así mismo, la SDB representa un método novedoso que revoluciona el campo de la topografía hidrográfica.

La batimetría óptica está respaldada por el principio de que la cantidad total de energía radiativa reflejada a partir de una columna de agua está en función de la profundidad, el espectro de la banda azul y verde tienen gran capacidad de penetración del agua (Gao, 2009). Uno de los modelos más utilizados en el ámbito de la SDB es el desarrollado por (Stumpf et al. 2003); este método resalta una solución empírica mediante una relación matemática de reflectancia, el algoritmo establece la correspondencia lineal entre la reflectancia de dos bandas (azul y verde); a medida que aumenta la profundidad del agua, los valores de reflectancia de la banda con mayor absorción disminuirán proporcionalmente más rápido que el valor de reflectancia de la banda con menor absorción (Jagalingam et al. 2015).

Las imágenes Landsat, principalmente, han aportado en gran medida al desarrollo de este este método, tanto así, que organizaciones internacionales como La Administración Nacional Oceánica y Atmosférica (NOAA) utilizan esta técnica para la actualización de cartas de navegación y como herramienta de reconocimiento para misiones hidrográficas (Rocchio, 2016), del mismo modo, la Organización Hidrográfica Internacional (IHO) recomienda el uso de la SDB como técnica complementaria para la estimación de profundidades e incluye el método en manuales de temas hidrográficos (IHO-IOC, 2019).

\section{Aplicaciones en el nivel mundial y regional}

La SDB se ha utilizado en diferentes lugares en el nivel mundial, por ejemplo (Benny \& Dawson, 1983) utilizaron imágenes de Landsat 3 MSS y datos in situ para determinar líneas de contorno de profundidad en una región al extremo norte del mar Rojo en Egipto; de igual manera, (Ibrahim \& Cracknell, 1990) utilizaron imágenes ópticas del sensor Landsat 3 MSS, determinaron la profundidad en las aguas costeras de la isla de Penang en Malasia; otro ejemplo, es la SDB realizada en la costa norte de Cape Ann, Massachusetts (EE. UU.), 
también en la región de Escravos que incluye la zona costera al suroeste del delta del río Niger (Nigeria) y el área de Big Creek ubicada en la costa sureste de Belice (Pe'eri et al. 2014); otro caso, corresponde a (Jagalingam et al. 2015) que utilizaron imágenes Landsat para cartografiar de la batimetría en la costa suroeste de la India; del mismo modo, (Deng et al. 2008) emplearon imágenes multiespectrales de diferentes sensores remotos ópticos y datos de batimetría in situ para determinar la profundidad en el estuario de Beilun en China; otro ejemplo, es el de (Putri et al. 2018) donde utilizaron imágenes multiespectrales y datos de batimetría in situ al derivar la SDB en la zona costera de Bangsring en Indonesia; (Kimeli et al. 2018) determinaron la SDB del canal portuario de Mombasa en Kenia mediante cartas náuticas e imágenes Landsat; como último ejemplo, (Knudby et al. 2016) evaluaron la aplicabilidad de las imágenes Landsat en la determinación de la SDB en "Boundary bay" en límites entre Canadá y EE. UU. en el Pacífico norte.

En el nivel regional (Ariza \& Ramírez, 2014) utilizaron imágenes Landsat 7 ETM + y datos de campo para determinar un modelo batimétrico en el monitoreo de arrecifes de coral en el archipiélago de San Andrés islas, Caribe colombiano; de igual manera, en el año 2018 se presentó los avances de La Comisión Hidrográfica Mesoamericana y del mar Caribe (MACHC) frente al mapeo batimétrico a través de sensores remotos ópticos en tres zonas, que incluyen a la Isla Grande en Cartagena Colombia (Goodrich, 2018); Estos referentes académicos denotan un antecedente en la aplicación de la SDB frente a los métodos tradicionales.

El principal objetivo de este estudio fue determinar la aplicabilidad de la SDB en aguas poco profundas en el sector conocido como "Punta Luna" en Bahía Solano, Colombia; este trabajo se presenta como un primer ejercicio en la zona y como una técnica complementaria que incorpora el procesamiento digital de imágenes de satélite, el manejo de datos batimétricos colectados in situ, la aplicación del modelo de Stumpf, la evaluación de la precisión en términos de ajuste del modelo SDB y la evaluación de la calidad de la SBD frente a estándares hidrográficos internacionales.

\section{MATERIALES Y METODOS}

\section{Área de estudio}

Se localiza al noreste del casco urbano de Ciudad Mutis sobre el litoral Pacífico colombiano del municipio de Bahía Solano, sector conocido localmente como "Punta Luna", en las coordenadas geográficas $6^{\circ}$ 16 ' 18.93 ' ' y $6^{\circ} 14^{\prime} 51.724$ ' ' N y los $77^{\circ} 24^{\prime} 20.26^{\prime \prime}$ y $77^{\circ} 23^{\prime} 10.968^{\prime}$ ' W (Fig. 1), posee un área aproximada de $1.72 \mathrm{~km}^{2}$ sobre el entorno marino. Esta zona se caracteriza por presentar 


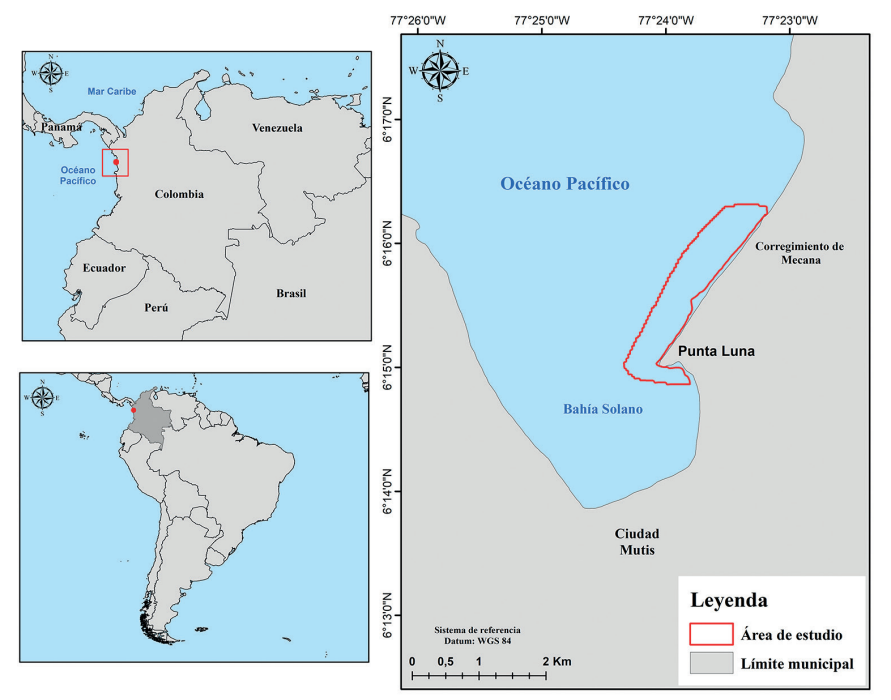

Fig. 1. Área de estudio. Sector "Punta Luna", municipio de bahía Solano - Colombia

Fig. 1. Study area. Punta Luna sector, municipality of Bahía Solano - Colombia

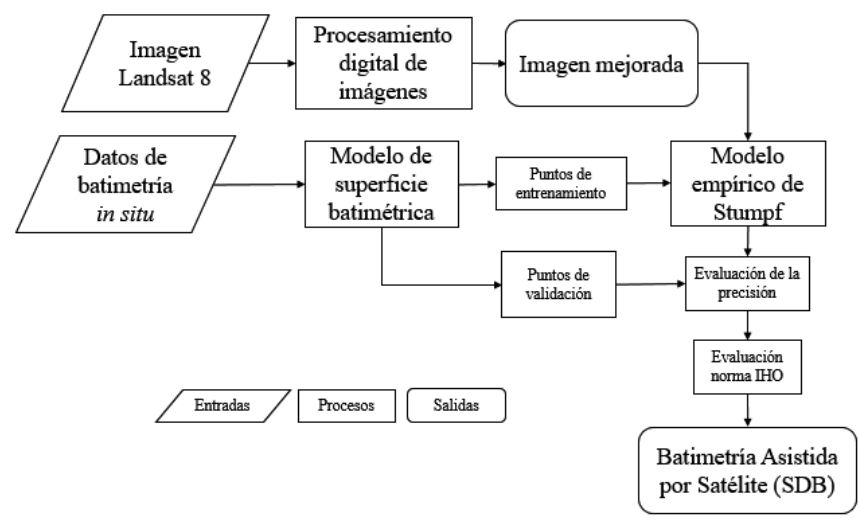

Fig. 2. Diagrama de flujo para la estimación de SBD Fig. 2. Flowchart for SDB estimate

diferentes paisajes que incluye planicies marinas, también, una alta dinámica mareal, precipitación promedio anual de $5000 \mathrm{~mm} \mathrm{y}$ una temperatura promedio anual de $26.3^{\circ} \mathrm{C}$ (Pierini \& Rodríguez, 2014).

Dentro del proceso metodológico (Fig. 2) se incorpora un flujo de trabajo para la obtención de la SDB que involucra pasos que siguen la lógica del proceso según lo recomendado por (Gao, 2009); para el manejo y procesamiento de datos se utilizó el software Arcgis 10.6 (ESRI, 2018).

\section{Imagen Landsat 8}

Se utilizó una imagen multiespectral del satélite Landsat 8, sensor OLI (Operational Land Imager) del 23 de enero de 2017 con resolución espacial de $30 \mathrm{~m}$ en bandas utilizadas (Azul, Verde y NIR), fue obtenida del centro de descarga de imágenes Landsat del Servicio Geológico de los Estados Unidos, a través de la plataforma web https://earthexplorer.usgs.gov/ (USGS, 2017) (Cuadro 1). Según Tang \& Mahmud, (2018) las bandas que generalmente se utilizan para el mapeo batimétrico están por el rango espectral de $(0.45-0.52 \mu \mathrm{m}) \mathrm{y}$ 
$(0.53-0.59 \mu \mathrm{m})$ correspondiente a la longitud de onda de la banda azul y verde del Landsat 8.

Procesamiento digital de imágenes

\section{Corrección atmosférica}

Se realizó la conversión de los valores de radiancia de los números digitales (ND) a unidades físicas de reflectancia ToA (Top of Atmosphere); se utilizó, en particular, las bandas correspondientes al rango del azul y verde, se empleó los parámetros propuestos por (Ariza, 2013; USGS, 2018) (Ecuación 1); este paso previo se realizó con el fin de eliminar los efectos atmosféricos de los aerosoles y la radiancia intrínseca al momento de la captura de la imagen, paso previo como parte del mejoramiento de la calidad de las imágenes (Aguilar et al. 2015).

$$
\boldsymbol{P}_{\lambda}=\frac{(M \rho * Q c a l+A \rho)}{\operatorname{Sin}\left(\emptyset_{\mathrm{se}}\right)}
$$

Donde:

$\mathrm{P}_{\lambda=}$ Reflectancia ToA

$\mathrm{M} \rho=$ Factor multiplicativo de escalado Qcal $=$ Valores de numero digitales (ND)

A $\rho=$ Factor aditivo de escalado $\varnothing_{\mathrm{se}}=$ Ángulo de elevación solar

\section{Ajuste del reflejo solar}

Adicionalmente, se realizó la corrección de la reflexión especular de la superficie del agua, según lo propuesto por (Hedley et al. 2005) (ecuación 2); en este proceso se utilizó la banda infrarroja (NIR); el ajuste se realizó para eliminar el efecto del reflejo de luz solar que pueda afectar los valores de ND para la predicción de la SDB en zonas de aguas poco profundas.

$$
\underline{R_{i}^{\prime}=R_{i}-b_{i}\left(R_{N I R}-M i n_{N I R}\right)}
$$

Donde:

$\mathrm{R}^{\prime}{ }_{\mathrm{i}}=$ Corrección del reflejo solar

$\mathrm{R}_{\mathrm{i}}=$ Valor del píxel de la banda (azul y verde)

$\mathrm{b}_{\mathrm{i}}=$ Pendiente de regresión

$\mathrm{R}_{\mathrm{NIR}}-\mathrm{Min}_{\mathrm{NIR}}=$ diferencia entre el píxel NIR (Infrarrojo cercano)

\section{Datos de batimetría in situ}

El levantamiento batimétrico se efectuó en el mes noviembre del año 2016 en aguas poco profundas en la zona de estudio, fue realizado por la 
Dirección General Marítima (Dimar) el método empleado fue el tradicional con el uso de una ecosonda monohaz (SBES) portátil de referencia HIDROTAC II (Fig. 3), instalada en una embarcación tipo lancha; del mismo modo, se utilizó receptores GNSS diferenciales los cuales siguieron una trayectoria de levantamiento.

Se obtuvo un total de 58082 puntos $X Y Z$, la profundidad registrada estuvo entre $0 \pm 15 \mathrm{~m}$; los datos se procesaron con apoyo del software CARIS HYPS ${ }^{\mathrm{TM}}$ versión 4.5 en oficina de levantamientos hidrográficos del Centro de Investigaciones Oceanográficas e Hidrográficas del Pacífico - CCCP; adicionalmente, se utilizó como referencia la carta náutica $\mathrm{N} .^{\circ} 710$ de la bahía interior de bahía Solano a escala 1:10.000, realizada por el Servicio Hidrográfico Nacional de Colombia (CIOH, 2017).

\section{Modelo de superficie batimétrica}

Con la información de la nube de puntos XYZ de la batimetría en in situ, se generó una superficie batimétrica tipo raster con tamaño de píxel de $30 \mathrm{~m}$, el

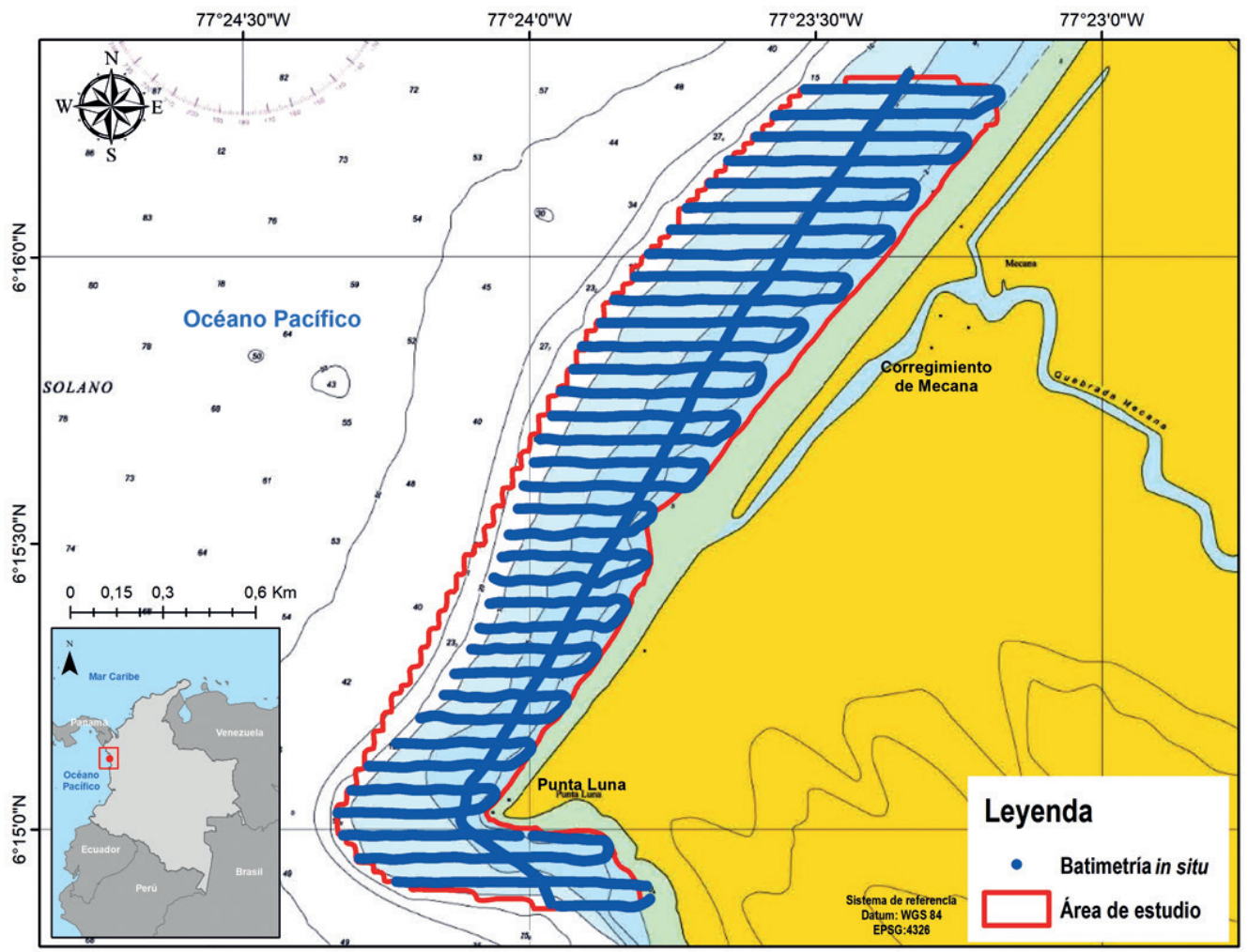

Fig. 3. Levantamiento batimétrico in situ con el método tradicional SBES Fig 3. In situ bathymetric survey using the traditional SBES method 
proceso se llevó a cabo con las herramientas de análisis 3D de Arcgis, adicionalmente, se seleccionó una muestra de 100 puntos aleatorios donde se extrajo la información de profundidades in situ para el ajuste inicial del modelo empírico, la muestra de profundidades se utilizó en el proceso de regresión lineal, aunque no se realizó un muestreo estadístico como tal, se procuró que los puntos fueran distribuidos homogéneamente y no saturaran los valores de regresión.

\section{Estimación de la SDB - Modelo Em- pírico de Stumpf}

Se aplicó el modelo empírico de (Stumpf et al.2003), (Ecuación 3). Este algoritmo utiliza la transformación del cociente entre la banda azul y verde la cual determina la batimetría relativa del fondo marino; este procedimiento permite estimarla eficientemente en aguas poco profundas de zonas costeras (Jagalingam et al. 2015). Se determinó las variables $\mathrm{m}_{1} \mathrm{y} \mathrm{m}_{0}$ por medio de una regresión lineal con los puntos de profundidad $(\mathrm{n}=100)$.

$$
Z=m_{1} \frac{\ln \left(n R_{w}\left(\lambda_{i}\right)\right)}{\ln \left(n R_{w}\left(\lambda_{j}\right)\right)}-m_{0}
$$

Donde:

$\mathrm{Z}=$ Valor derivado de la profundidad $\mathrm{R}_{\mathrm{w}}=$ Reflectancia ToA observada de la banda azul $\mathrm{i}$ y verde $\mathrm{j}$

$\mathrm{m}_{1}=$ Constante para escalar la relación de la profundidad

$\mathrm{m}_{0}=$ Desplazamiento de la profundidad $\mathrm{n}=$ Valor constante

\section{Evaluación de la precisión}

Se crearon 100 puntos aleatorios distribuidos homogéneamente sobre la SDB para contar con el mismo número de puntos en el análisis de precisión, se extrajo el valor de profundidad sobre el resultado SDB, se procedió a realizar la comparación de las profundidades observadas (in situ) vs. las estimadas por el modelo (SDB); se determinó el Error Medio Cuadrático (RMSE), Error Medio Absoluto (MAE) y el coeficiente de determinación de ajuste $\left(\mathrm{R}^{2}\right)$ como parámetros estadísticos de evaluación, se utilizó las ecuaciones expuestas en (Tang \& Mahmud, 2018) (Ecuación 4 y 5); según (Gao, 2009) estos parámetros de evaluación se utilizan como una medida de precisión de la SDB estimada con imágenes ópticas; se realizó una gráfico donde se comparó los valores observados vs. estimados; adicionalmente, se realizó un perfil de la sección central, que determina la incertidumbre del modelo SDB frente a la profundidad real y la distancia en metros aguas adentro.

$$
\begin{aligned}
& M A E=\frac{1}{n} \sum_{j=1}^{1}\left|Z_{S D B}-Z_{F B}\right| \\
& R M S E=\sqrt{\frac{1}{n} \sum_{j=1}^{1}\left(Z_{S D B}-Z_{F B}\right)^{2}}
\end{aligned}
$$

Donde:

$\mathrm{n}=$ Número de puntos de la batimetría $\mathrm{Z}_{\mathrm{SDB}}=$ Valor de profundidad de la SDB $(n=100)$ 
$\mathrm{Z}_{\mathrm{FB}}=$ Valor profundidad batimetría en in situ $(\mathrm{n}=100)$

\section{Evaluación estándar IHO S-44}

Como parte de la evaluación de calidad de los datos derivados, se transformó la SDB raster a una malla de puntos XYZ, después, se calculó para ambas mallas la Incertidumbre Vertical Total (TVU) (Ecuación 6) del estándar de la Organización Hidrográfica Internacional (IHO) en la publicación N. ${ }^{\circ}$ S-44 para levantamientos hidrográficos (OIH, 2008); este protocolo tiene por objeto calcular las incertidumbres que se presentan en la toma de datos de profundidad, la TVU define categorías denominadas "Órdenes" específicamente para detectar "rasgos", referentes a objetos en el fondo marino sean naturales o no, que afecten la seguridad de la navegación, el estándar contempla una medida de incertidumbre con un nivel de confianza que corresponde al 95\%.

$$
\pm \sqrt{a^{2}+(b x d)^{2}}
$$

Donde:

$\mathrm{a}=$ Porción de la incertidumbre que no varía con la profundidad

$b=$ Coeficiente que varía con la profundidad

$\mathrm{d}=$ Es la profundidad

\section{RESULTADOS}

Se obtuvo dos superficies batimétricas a resolución de $30 \mathrm{~m}$ para los datos in situ y SDB, se encontró que el área de estudio morfológicamente presentó un fondo marino prolongado a través de terrazas marinas; de igual manera, la superficie SDB evidenció una profundidad promedio de $6.03 \mathrm{~m}$, el valor mínimo de SDB correspondió a -5.53 m (Cuadro 2) este valor negativo correspondió a zonas con procesos de acreción.

Cuadro 2. Estadísticas descriptivas de superficies batimétricas

Table 2. Descriptive statistics of bathymetric surface

\begin{tabular}{ccc}
\hline Parámetro & $\begin{array}{c}\text { Superficie } \\
\text { in situ }(\mathbf{m})\end{array}$ & $\begin{array}{c}\text { SDB } \\
(\mathbf{m})\end{array}$ \\
\hline Mínimo & 0.23 & -5.53 \\
Máximo & 15 & 11.66 \\
Promedio & $6.29 \pm 3.97$ & $6.03 \pm 2.82$ \\
\hline
\end{tabular}

En la visualización, se puede observar que la SDB asemeja en gran medida el comportamiento de la superficie derivada con datos in situ (Fig. 4); adicionalmente, se observó que la SDB presentó valores negativos sobre la línea de costa, además, de un valor de cero $(0)$ correspondiente a la aparición de un rasgo sobre la parte central al noreste que no se visualiza en los datos in situ.

\section{Evaluación del modelo empírico de Stumpf}

Dentro de la comparación de las superficies batimétricas derivadas, se evidenció que la SDB obtuvo un promedio de profundidad de 6.30 

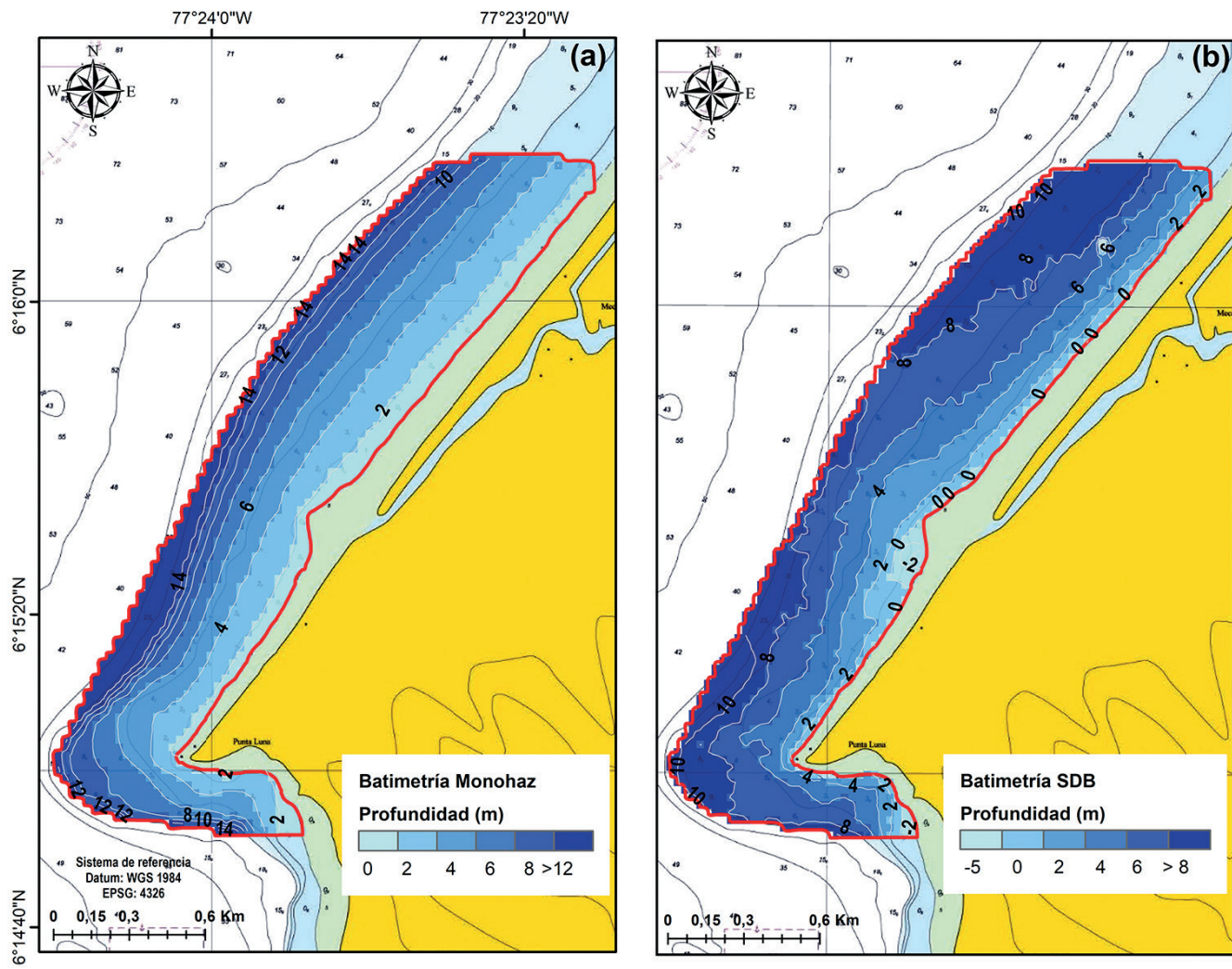

Fig. 4. (a) Modelo de superficie batimétrica; (b) Batimetría derivada por satélite (SDB) Fig. 4. (a) Bathymetric Surface model, (b) Satellite-Derived Bathymetry (SDB)

m (Cuadro 3); la muestra de pun- del modelo determinó los parámetos $(n=100)$ fue acorde y no presen- tros de ajuste $m_{1} \mathrm{y} \mathrm{m}_{0}$; el valor del tó saturación en la regresión lineal RMSE y MAE fue de 1.49 y $2.22 \mathrm{~m}$ que alcanza un $\mathrm{R}^{2}$ de 0.71 ; el ajuste respectivamente.

Cuadro 3. Parámetros estadísticos para la evaluación de la precisión

Table 3. Statistics parameters for accuracy assessment

\begin{tabular}{cccccc}
\hline Parámetro & $\begin{array}{c}\text { Batimetría } \\
\text { in situ (m) }\end{array}$ & $\begin{array}{c}\text { SDB } \\
(\mathbf{m})\end{array}$ & \multicolumn{3}{c}{ Parámetros de ajuste } \\
\cline { 3 - 6 } Mínimo & 0.66 & -3.75 & 0.71 & 267.96 & 292.1 \\
Máximo & 14.98 & 10.89 & & & \\
Promedio & $6.98 \pm 4.20$ & $6.30 \pm 2.67$ & & & \\
RMSE & \multicolumn{2}{c}{$1.49 \mathrm{~m}$} & & & \\
MAE & $2.22 \mathrm{~m}$ & & & \\
\cline { 1 - 2 }
\end{tabular}

Se obtuvo la gráfica de cotejo de ambas medidas, se comparó las profundidades observadas $v s$. las estimadas desde el modelo SDB (Fig. 5); el perfil de profundidad alcanzó una $\mathrm{R}^{2}=0.80$, se observó que la SDB 

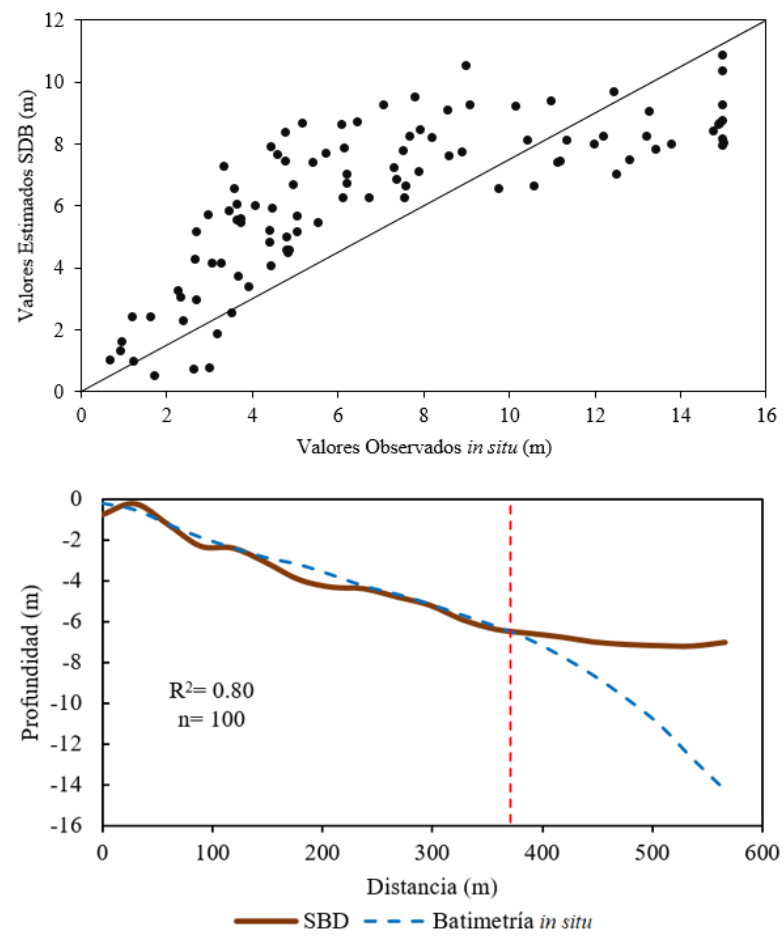

Fig. 5. (Superior) Profundidad de valores observados y estimados. (Inferior) Perfil batimétrico de incertidumbre entre datos in situ y SDB

Fig. 5. (Top) Depth of observed and estimated values. (Bottom) Bathymetric profile of uncertainty between data in situ and SDB

alcanza la mayor incertidumbre a los $7 \mathrm{~m}$ de profundidad.

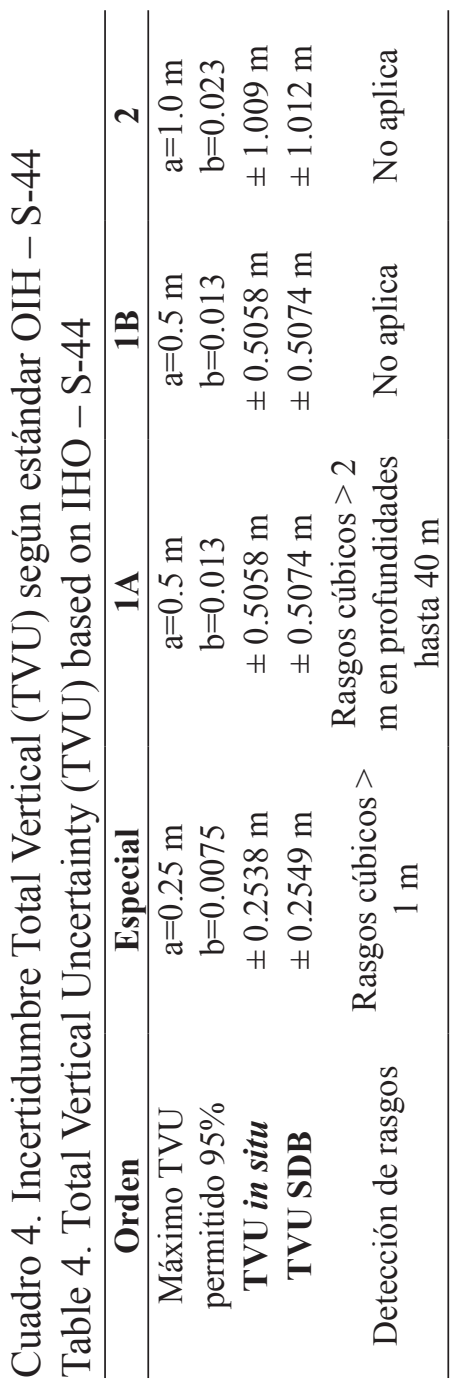

\section{Estándar IHO-S44}

Se determinó el valor de incertidumbre máxima TVU permitida en ambas superficies (Cuadro 4); se observó que los valores de TVU para la SDB son ligeramente mayores a los de la batimetría in situ para las diferentes categorías del estándar S-44 en la detección de rasgos.

De igual manera, los puntos de profundidad que cumplen la categoría de Orden Espacial corresponden el 75.10 y $51.17 \%$ para datos in situ y la SDB respectivamente (Cuadro 5); el valor de rechazo más alto de TVU correspondió para el Orden 2 de la SDB con el $73.40 \%$. 


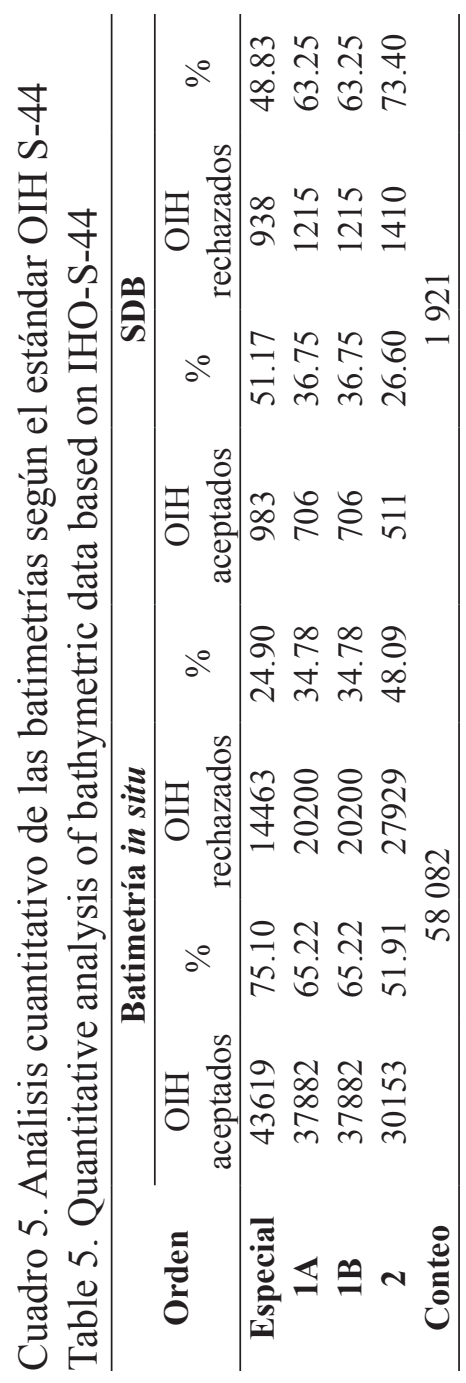

\section{DISCUSIÓN}

Las imágenes Landsat 8 utilizadas en este estudio presentaron una buena calidad y no evidenciaron factores ambientales que pudiesen alterar los posteriores análisis; según (Jégat et al. 2016; IHO-IOC, 2019) las imágenes Landsat son las más utilizadas para derivar productos SDB. Se utilizan desde varias décadas debido a la fácil adquisición y libre disposición para la comunidad científica (Pacheco et al. 2016; Kimeli et al. 2018).

La determinación de la SDB a través del método empírico de Stumpf obtuvo un $\mathrm{R}^{2}=0.80$ respecto los datos tomados in situ; el resultado de este estudio presentó mejor ajuste frente a los reportados por (Setiawan et al. 2017; Ashphaq, 2018; Ekpa \& Ojinnaka, 2018; Tang \& Mahmud, 2018); por otro lado, presenta gran similitud al valor del ajuste para análisis reportados por (Jagalingam et al. 2015; Pushparaj \& Hegde 2016; Kimeli et al. 2018; Putri et al. 2018); del mismo modo, se pudo evidenciar que el valor de la reflectancia de la banda azul y verde aumenta cuando las aguas se vuelven poco profundas; por ende, se presenta una relación inversamente proporcional en relación a la profundidad (Gao, 2009); otro aspecto a considerar es incluir en el análisis la banda 1 (Coastal/Aerosol) del Landsat 8, ya que cumple con el rango espectral de aplicación y se ha visto que mejora sustancialmente la estimación de la profundidad en aguas costeras (Vinayaraj et al. 2016; Kabiri, 2017).

La imagen satelital utilizada en este estudio, presentó dos meses de diferencia en comparación a los datos levantados in situ, los valores negativos en la SDB pueden evidenciar posibles fenómenos dinámicos de acreción de sedimentos, estos datos pueden servir en gran medida para el seguimiento de la 
morfología y dinámica costera (Pacheco et al. 2016). Cabe mencionar que la SDB proporciona una oportunidad para la investigación científica en varias líneas como, la planificación espacial marina, gestión costera, acuicultura, geología marina, la ingeniería, ciencias biológicas y marinas entre otros propósitos (Jonas, 2018; Caballero \& Stumpf, 2020). La SDB también contempla apoyo a las autoridades regionales en tareas comunes de línea base, delimitación, análisis de cambios del fondo marino, cartografía batimétrica para brindar seguridad en la navegación (Politi et al. 2019).

El RMSE y MAE alcanzaron un valor de 1.49 y $2.22 \mathrm{~m}$ respectivamente; el RMSE fue similar al reportado por (Kimeli et al. 2018) pero superiores a los registrados por (Jagalingam et al. 2015); el MAE por su parte fue superior al reportado por (Tang \& Mahmud, 2018); otras SDB determinadas con imágenes de mediana resolución como las del Sentinel 2 reportaron valores similares (Chybicki, 2017; Evagorou et al. 2019); las variaciones temporales y condiciones ambientales no favorables en la imagen son factores que pueden aumentar el error de la estimación de la SDB (Bramante et al. 2013; Pushparaj \& Hegde, 2016; Evagorou et al. 2019).

Este estudio demostró que la SDB con imágenes Landsat 8 alcanzó a cartografiar una profundidad efectiva de hasta $\pm 7 \mathrm{~m}$, resultados superiores al reportado por (Jagalingam et al. 2015; Najhan et al. 2017; Kimeli et al.
2018); el perfil longitudinal de este trabajo demostró que el modelo se vuelve constante y no correlacionado en profundidades superiores a $\operatorname{los} 7 \mathrm{~m}$, lo cual aumenta en gran medida la incertidumbre de los datos (Chybicki, 2017); de igual manera, Gao, (2009) afirma que se puede cartografiar la SDB con una precisión hasta $7.5 \mathrm{~m}$ de profundidad y que algunos factores como la turbidez, los materiales del fondo y las características propias de la imagen pueden afectar en gran medida la estimación de esta variable; sumado a esto Pe'eri et al. (2014) menciona que las condiciones ambientales como la cobertura de nubes y el reflejo del sol sobre la lámina de agua son otros factores que pueden generar incertidumbre en la determinación de la profundidad con la SDB.

En la zona de estudio se han realizado mediciones en la concentración de los Sólidos Suspendidos Totales (SST), los resultados han demostrado que en épocas de lluvia la concentración de SST aumenta debido al transporte de sedimentos y procesos de erosión de las fuentes hídricas que desembocan a la bahía, lo cual incide negativamente en la penetración de la luz al fondo marino (Vivas-Aguas et al. 2012); esto puede ser un factor clave para realizar la SDB en una época determinada del año (Tang \& Mahmud, 2018). Es necesario profundizar en el estudio de las propiedades ópticas inherentes del océano (OIPs) ya que a través de elementos y partículas 
suspendidas en el océano se reflejan indicadores de la calidad del agua y estos puede afectar negativamente la respuesta espectral del fondo marino capturada por sensores (Dierssen et al. 2003).

La evaluación de las batimetrías estuvo acorde a los valores permitidos para la TVU en la norma OIH - S44 para levantamientos hidrográficos, de igual manera, el $51.17 \%$ de las profundidades de la SDB alcanzó la categoría de Orden Especial, este orden es el más riguroso dentro de la clasificación de los levantamientos hidrográficos de la norma S-44 y es de exclusivo interés en zonas donde se presentan atraques, puertos y áreas críticas para la navegación (OIH, 2008). Aunque, la $\mathrm{OIH}$ no tiene un estándar específico para evaluar la calidad del levantamiento batimétrico con la SDB, surge una oportunidad de generar protocolos y adoptarlos a productos para la navegación (Mavraeidopoulos et al. 2017). De igual manera, diferentes servicios hidrográficos en el nivel mundial han utilizado la SBD como complemento dentro de la generación de información batimétrica de reconocimiento y generación de productos hidrográficos de bajo costo (Jégat et al. 2016; Rocchio, 2016; Chéiner et al. 2018).

\section{CONCLUSIONES}

El presente estudio reflejó una aproximación a la cartografía batimétrica que utiliza imágenes multiespectrales de mediana resolución del Landsat 8 en un sector de Bahía Solano en Colombia; además, se presenta como una oportunidad para desarrollar temas de investigación que involucren el Manejo Integrado de Zonas Costeras - MIZC, temas que son de especial interés para las ciencias marinas y la ingeniería; el uso de la SDB puede verse como un complemento para adquirir datos de profundidades en zonas costeras donde no existan, esto debido a los bajos costos de adquisición de las imágenes de satélite y el rápido procesamiento al utilizar Teledetección y SIG; la SDB puede ser manejada como una herramienta complementaria de apoyo para las diferentes oficinas hidrográficas que requieran información batimétrica de lugares específicos y en la planificación de futuras misiones de levantamiento hidrográfico.

Por otro lado, se recomienda el uso de otras imágenes de satélite de sensores ópticos de mayor resolución espacial para derivar SDB en aguas poco profundas, como también, la comparación de otros modelos empleados en la literatura para determinar el rendimiento de la SDB con diferentes enfoques metodológicos.

\section{AGRADECIMIENTOS}

A la Dirección General Marítima de Colombia, en especial al Área de Manejo Integrado de Zona Costera 
del Centro de Investigaciones Oceanográficas e Hidrográficas del Pacífico CCCP; a la oficina de Levantamientos Hidrográficos del CCCP en Tumaco, por la autorización en el uso de datos batimétricos oficiales y a los evaluadores anónimos por las recomendaciones.

\section{REFERENCIAS}

Aguilar, H., Mora, R. \& Vargas, C. (2015). Metodología para la corrección atmosférica deimágenes Aster, Rapideye, Spot 2 y Landsat 8 con el módulo Flaash del software Envi. Rev. Geogr. Am. Cent., 2(53), 39-59. https://doi. org/10.15359/rgac.2-53.2

Ariza, A. (2013). Descripción y corrección de productos Landsat $8 \mathrm{LDCM}$ (Landsat Data Continuity Mission) Versión 1.0. Centro de Investigación y Desarrollo - CIAF. Instituto Geográfico Agustín Codazzi. www.un-spider.org/sites/default/files/LDCM-L8.R1.pdf

Ariza, A. \& Ramírez, H. M. (2014). Modelo batimétrico derivado de imágenes Landsat ETM + en zonas de arrecifes tropicales. Rev. Cartogr., 90, 43-58.

Ashphaq, M. (2018). Bathymetry estimation in turbid water using SENTINEL 2 image. https://www.adv-geosci. net/45/397/2019/adgeo-45-397-2019.pdf

Benny, A. H. \& Dawson, G. J. (1983). Satellite Imagery as an Aid to Bathymetric Charting in the Red Sea. CARTOGR J., 20(1), 5-16. http://doi.org/10.1179/ caj.1983.20.1.5

Bramante, J. F., Raju, D. K. \& Sin, T. M. (2013). Multispectral derivation of bathymetry in Singapore's shallow, turbid waters. Int. J. Remote Sens., 34(6), 2070-2088. http://doi.org/10.1080/014 31161.2012.734934
Caballero, I. \& Stumpf, R. P. (2020). Atmospheric correction for satellite-derived bathymetry in the Caribbean waters : from a single image to multi-temporal approaches using Sentinel-2A/B. Opt. Express, 28(8), 11742-11766. http:// doi.org/10.1364/OE.390316

$\mathrm{CIOH}$. Centro de Investigaciones Oceanográficas e Hidrográficas del Caribe. (2017). Carta Hidrográfica 710, bahía interior de Solano Escala 1:10.000. https://www.cioh.org.co/index.php/es/ cartas-nauticas.html

Chéiner, R., Faucher, M. A. \& Ahola, R. (2018). Satellite-Derived Bathymetry for Improving Canadian Hydrographic Service Charts. ISPRS Int. J. Geo-Inf., 7(306), 2-15. http://doi.org/10.3390/ ijgi7080306

Chybicki, A. (2017). Mapping south baltic near-shore bathymetry using sentinel-2 observations. Pol. Marit. Res., 24(3), 15-25. https://doi.org/10.1515/ pomr-2017-0086

Deng, Z., Ji, M. \& Zhang, Z. (2008). Mapping bathymetry from multi-source remote sensing images: a case study in the beilun estuary, guangxi, China. Int. Arch. Photogramm. Remote Sens. Spat. Inf. Sci., 37, 1321-1326. https://www.isprs. org/proceedings/XXXVII/congress/8_ pdf/13_ThS-19/05.pdf

Dierssen, H. M., Zimmerman, R. C. Leathers, R. A. Downes, T. V. \& Davis, C. O. (2003). Ocean color remote sensing of seagrass and bathymetry in the Bahamas Banks by high-resolution airborne imagery. Limnol. Oceanogr., 48, 444-455. https://doi.org/10.4319/ lo.2003.48.1 part 2.0444

Ekpa, A. U. \& Ojinnaka, O. C. (2018). Estimating bathymetry of cross river in Nigeria using remote sensing technique. SSRG Int. J. Geoinf. Geol. Sci., 5(3), 1-15. 
https://doi.org/10.14445/23939206/ IJGGS-V5I3P101

ESRI. (2018). ArcGIS Desktop: Release 10.6. EE. UU.: Environmental Systems Research Institute. https://desktop.arcgis.com/es/

Evagorou, E., Mettas, C. Agapiou, A. Themistocleous, K. \& Hadjimitsis, D. (2019). Bathymetric maps from multi-temporal analysis of Sentinel-2 data: the case study of Limassol, Cyprus. Adv. Geosci., 45, 397-407. https://doi. org/10.5194/adgeo-45-397-2019

Gao, J. (2009). Bathymetric mapping by means of remote sensing: Methods, accuracy and limitations. Prog. Phys. Geogr., 33(1), 103-116. https://doi. org/10.1177/0309133309105657

Goodrich, K. (2018). Machine Learning Applications for Satellite Derived Bathymetry. Colombia: GEBCO. https:// iho.int $/ \mathrm{mtg}$ _docs/rhc/MACHC/MACHC19/MACHC19-06.7-TCarta.pdf

Hedley, J., Harborne, A. \& Mumby, P. (2005). Simple and robust removal of sun glint for mapping shallow-water benthos Simple and robust removal of sun glint for mapping shallow-water. Int. J. Remote Sens., 26(10), 2107-2112. https:// doi.org/10.1080/01431160500034086

Ibrahim, M. \& Cracknell, A. P. (1990). Cover Bathymetry using Landsat MSS data of Penang Island in Malaysia. Int. J. Remote Sens., 11(4), 557-559. http://doi. org/10.1080/01431169008955040

IHO-IOC. International Hydrographic Organization - Intergovernmental Oceanographic Commission (2019). The IHO-IOC GEBCO CookBook. Francia: OHI Publication B-11, IOC Manuals and Guides 63. International Hydrographic Organization, Intergovernmental Oceanographic Commission. https:// www.star.nesdis.noaa.gov/socd/lsa/ GEBCO_Cookbook/
Jagalingam, P., Akshaya, B. J. \& Hegde, A. V. (2015). Bathymetry mapping using landsat 8 satellite imagery. Procedia Eng., 116(1), 560-566. https://doi.org/10.1016/j.proeng.2015.08.326

Jégat, V., Pe'eri, S. Freire, R. Klemm, A. \& Nyberg, J. (2016, mayo). Satellite-Derived Bathymetry: Performance and Production. Canadian Hydrographic Conference, Halifax, NS.

Jonas, M. (2018, junio). Why is satellite derived bathymetry needed? SDB Day, User and Technology Forum, Munich, Alemania.

Kabiri, K. (2017). Accuracy assessment of near-shore bathymetry information retrieved from Landsat-8 imagery. Earth Sci. Inform., 10, 235-245. http://doi. org/10.1007/s12145-017-0293-7

Kimeli, A., Thoya, P. Ngisiang, N. Ong, H. \& Magori, C. (2018). Satellite-derived bathymetry: A case study of Mombasa Port Channel and its approaches, Kenya. West. Indian Ocean J. Mar. Sci., 17(2), 93-102. http://dx.doi. org/10.4314/wiojms.v17i2.8

Knudby, A., Ahmad, S. K. \& Ilori, C. (2016). The Potential for Landsat-Based Bathymetry in Canada. Can. J. Remote. Sens., 42, 367-378. http://doi.org/10.1 080/07038992.2016.1177452

Lyzenga, D. (1978). Passive remote sensing techniques for mapping water depth and bottom features. Appl. Opt., 17(3), 379-383. http://doi.org/10.1364/ AO.17.000379

Lyzenga, D. (1985). Shallow-water bathymetry using combined lidar and passive multispectral scanner data. Int. J. Remote Sens., 6(1), 115-125. https://www.tandfonline.com/doi/ abs/10.1080/01431168508948428

Mavraeidopoulos, A., Navy, H. Pallikaris, A. Academy, H. N. \& Oikonomou, E. (2017). Satellite derived bathymetry (SDB) and safety of navigation. Int. 
Hydrogra. Rev., 7-19. https://journals.lib.unb.ca/index.php/ihr/article/ view/26290

Najhan, S., Mohd, M. \& Rozaimi, H. (2017). Satellite-Derived Bathymetry: Accuracy assessment on depths derivation algorithm for shallow water area. Int. Arch. Photogramm., Remote Sens. Spat. Inform. Sci., 42, 159-164. https://doi.org/10.5194/ isprs-archives-XLII-4-W5-159-2017

OIH. Organización Hidrográfica Internacional. (2008). Normas de la OHI para los Levantamientos Hidrográficos. S-44 (5th ed.). Francia. Bureau Hidrográfico Internacional. http://bathyswath.com/sites/ default/files/documents/S-44_5S.pdf

Pacheco, A., Horta, J. Loureiro, C. \& Ferreira, Ó. (2016). Retrieval of nearshore bathymetry from Landsat 8 images: A tool for coastal monitoring in shallow waters. Remote Sens. Environ, 159, 102-116. https://doi.org/10.1016/j. rse.2014.12.004

Pe'eri, S., Parrish, C. Azuike, C. \& Armstrong, A. (2014). Satellite remote sensing as a reconnaissance tool for assessing nautical chart adequacy and completeness. Mar. Geod., 37(3), 37-41. https://doi.or g/10.1080/01490419.2014.902880

Philpot, W. (1989). Bathymetric mapping with passive multispectral imagery. Appl. Opt., 28(8), 1569-1578. http://doi. org/10.1364/AO.28.001569

Pierini, O. \& Rodríguez, A. (2014). Caracterización oceanográfica de la bahía de Solano. Boletín Cientifico CIOH., 32, 223-256. https://doi. org/10.26640/22159045.274

Polcyn, F. C., Brown, W. L. \& Sattinger, I. J. (1970). The Measurement of Water Depth by Remote Sensing Techniques. EE. UU.: The Institute of Science and Technology, The University of Michigan. https://pdfs.semanticscholar. org/59b2/a818598554825c7923bc3d51edd64d443f1d.pdf

Politi, E., Paterson, S. K. Scarrott, R. Tuohy, E. Mahony, C. O. \& Cámaro-garcía, W. C. A. (2019). Earth observation applications for coastal sustainability: potential and challenges for implementation. Anthropocene Coasts., 2(1), 306-329. https://doi.org/10.1139/anc-2018-0015

Pushparaj, J. \& Hegde, A. V. (2016). Estimation of bathymetry along the coast of Mangaluru using Landsat-8 imagery. Int. J. Ocean Climate Syst., 8(2), 71-83. https://doi. org $/ 10.1177 / 1759313116679672$

Putri, J. C. A., Fuad, M. A. Z. \& Asa'di, M. A. (2018). Bathymetry mapping using Landsat 8 multyspectral data of bangsring coastal area. Omni-Akuatika, 14(1), 54-61. https://doi. org/10.20884/1.oa.2018.14.1.368

Rocchio, L. E. P. (2016). Avoiding Rock Bottom: How Landsat Aids Nautical Charting. Landsat Sci., 71-77. https:// landsat.gsfc.nasa.gov/wp-content/ uploads/2016/08/Landsat_Improve_ Life_Bathymetry.pdf

Setiawan, K., Adawiah, S. Marini, Y. \& Winarso, G. (2017). Bathymetry data extraction analysis using Landsat 8 data. Int. J. Remote Sens. Earth Sci., 13(2), 79-86. https://doi.org/10.30536/j.ijreses.2016.v13.a2448

Stumpf, R. P., Holderied, K. \& Sinclair, M. (2003). Determination of water depth with high-resolution satellite imagery over variable bottom types. Limnol. Oceanogr, 48, 54-556. https://doi. org/10.4319/1o.2003.48.1_part_2.0547

Tang, K. K. W. \& Mahmud, M. R. (2018). Imagery-derived bathymetry in Strait of Johor's turbid waters using multispectral images. Int. Arch. Photogramm. Remote Sens. Spat. Inf. Sci, 
42, 139-145. https://doi.org/10.5194/ isprs-archives-XLII-4-W9-139-2018

USGS. U. S. Geological Survey. (2017). Imágen Multiespectral Landsat 8 OLI, ID LC80100562017023LGN01. EE. UU.: Landsat Data Continuity Mission. https://earthexplorer.usgs.gov/

USGS. U. S. Geological Survey. (2018). Landsat 8 (L8) Data Users Handbook - Version 3.0. EE. UU.: USGS. https:// prd-wret.s3-us-west-2.amazonaws. com/assets/palladium/production/ s3fs-public/atoms/files/LSDS-1574 L8_Data_Users_Handbook.pdf

Vinayaraj, P., Raghavan, V. \& Masumoto, S. (2016). Satellite-Derived Bathymetry using Adaptive Geographically Weighted Regression Model. Mar. Geod., 39(6), 458-478. http://doi.org/1 $0.1080 / 01490419.2016 .1245227$
Vivas-Aguas, L. J., Espinosa, J. Sánchez, B. Cadavid, P. Bautista, M. Quintero, J...\& Espinosa. (2012). Diagnóstico y Evaluación de la Calidad Ambiental Marina en el Caribe y Pacífico colombiano. Red de vigilancia para la conservación y protección de las aguas marinas y costeras de Colombia-REDCAM. Informe técnico 2011. Santa Marta: INVEMAR.

Warne, D. K. (1978). Landsat as an Aid in the Preparation of Hydrographic Charts. Photogramm Eng Remote Sensin., 44(8), 1011-1016. 